\title{
Alternative momentum concept for a quantum mechanical particle in a box
}

\author{
M. H. Al-Hashimi $\odot$ and U.-J. Wiese $\odot$ \\ Albert Einstein Center, Institute for Theoretical Physics, University of Bern, 3012 Bern, Switzerland
}

(Received 22 December 2020; revised 14 July 2021; accepted 15 September 2021; published 26 October 2021)

\begin{abstract}
For a particle in a box, the operator $\hat{p}=-i \partial_{x}$ is not self-adjoint. We provide an alternative construction of a momentum operator $\hat{p}_{R}+i \hat{p}_{I}$, which has two self-adjoint components $\hat{p}_{R}$ and $\hat{p}_{I}$. This leads to a description of momentum measurements performed on a particle that is strictly limited to the interior of a box of size $L$, which yields quantized momentum values $\pi n / L$ with $n \in \mathbb{Z}$.
\end{abstract}

DOI: 10.1103/PhysRevResearch.3.L042008

\section{INTRODUCTION}

Momentum is one of the most important physical observables. It generates translations in space and is thus conserved when the Hamiltonian is translation invariant. Many important quantum systems, including ultra-cold atoms in an optical box trap [1], quantum dots [2], and quantum billiards [3,4], phenomenological bag models for confined quarks and gluons [5-7], or regions of extra-dimensional space for domain wall fermions [8,9], are naturally modeled by impenetrable spatial boundaries which explicitly violate translation invariance. While this idealization allows the exclusion of irrelevant spatial regions with large potential energy, it is conceivable that extra-dimensional space literally ends at a boundary [10]. In all these cases, the standard quantum mechanical momentum does not represent a physical observable because the corresponding operator is not self-adjoint. In this letter, we develop an alternative concept for the quantum mechanical momentum which is applicable to a particle that is strictly confined inside a finite interval (see, also, Ref. [11]). The alternative concept naturally extends to higher-dimensional spaces with boundaries as well. As we will see, in contrast to the standard momentum, the alternative momentum concept leads to a discrete spectrum of allowed momenta in a finite volume.

In quantum mechanics physical observables are described by self-adjoint operators. The subtle differences between Hermiticity and self-adjointness, which arise because the Hilbert space is infinite-dimensional, were first understood by von Neumann [12]. In particular, in addition to Hermiticity, selfadjointness of an operator $\hat{A}$ requires that its domain $D(\hat{A})$ coincides with the domain $D\left(\hat{A}^{\dagger}\right)$ of its adjoint $\hat{A}^{\dagger}$ [13-15]. The domain of a differential operator is characterized by square-integrability conditions on derivatives of the wave functions. For a particle in a finite volume, boundary con-

Published by the American Physical Society under the terms of the Creative Commons Attribution 4.0 International license. Further distribution of this work must maintain attribution to the author(s) and the published article's title, journal citation, and DOI. ditions (characterized by self-adjoint extension parameters) further restrict the domain [16-18]. Only self-adjointness (and not Hermiticity alone) guarantees a real-valued spectrum of eigenvalues and an orthonormal set of corresponding eigenfunctions. This is crucial for the correct description of measurements that return the eigenvalues of the corresponding operator. It is well-known that, for a particle in a one-dimensional box, the operator $\hat{p}=-i \partial_{x}$ is not selfadjoint [19]. In this "simple" quantum mechanical problem [20-22], momentum measurements are thus nontrivial [23].

\section{STANDARD MOMENTUM MEASUREMENTS FOR A PARTICLE IN A BOX}

Let us first consider the standard momentum operator $\hat{p}=$ $-i \partial_{x}$ (in units where $\hbar=1$ ) for a quantum mechanical particle moving along the real axis, which acts in the Hilbert space $L^{2}(\mathbb{R})$ of square-integrable functions over the entire real axis $\mathbb{R}$. The operator $\hat{p}$ can act only on those wave functions $\Psi(x)$ that are differentiable at least once. In addition, the resulting wave function $-i \partial_{x} \Psi(x)$ should also be square integrable, such that $\hat{p}$ maps $\Psi(x)$ back into the Hilbert space $L^{2}(\mathbb{R})$. These restrictions define the domain $D(\hat{p}) \subset L^{2}(\mathbb{R})$ in which $\hat{p}$ acts. By definition, the Hermitean conjugate $\hat{p}^{\dagger}$ acts as $\left\langle\hat{p}^{\dagger} \chi \mid \Psi\right\rangle=\langle\chi \mid \hat{p} \Psi\rangle$. By partial integration one readily shows that $\hat{p}$ is Hermitean (or symmetric in mathematical parlance), i.e., $\hat{p}^{\dagger} \Psi=\hat{p} \Psi$ for $\Psi \in D(p)$. In addition, the two domains coincide, $D\left(\hat{p}^{\dagger}\right)=D(\hat{p})$, such that $\hat{p}$ is indeed self-adjoint. The eigenstates of the momentum operator are plane waves $\langle x \mid k\rangle=\exp (i k x)$ with a continuous momentum value $k \in \mathbb{R}$. Since they are not square-integrable, the momentum eigenstates do not belong to the Hilbert space, but are normalized to $\delta$-functions, $\left\langle k \mid k^{\prime}\right\rangle=2 \pi \delta\left(k-k^{\prime}\right)$.

When we restrict ourselves to the finite interval $\left[-\frac{L}{2}, \frac{L}{2}\right]$, the operator $\hat{p}=-i \partial_{x}$ is not self-adjoint, unless one imposes periodic boundary conditions on the probability density. This is unphysical in the present context, because we are interested in an interval with physically distinct endpoints. By partial integration one obtains

$$
\left\langle\hat{p}^{\dagger} \chi \mid \Psi\right\rangle=\langle\chi \mid \hat{p} \Psi\rangle=\langle\hat{p} \chi \mid \Psi\rangle-i\left[\chi(x)^{*} \Psi(x)\right]_{-L / 2}^{L / 2} .
$$


Hermiticity requires $\chi\left( \pm \frac{L}{2}\right)^{*} \Psi\left( \pm \frac{L}{2}\right)=0$. This can be achieved by restricting the domain $D(\hat{p})$ to those wave functions that obey $\Psi\left( \pm \frac{L}{2}\right)=0$ and whose derivative is square integrable at least once. Then $\chi\left( \pm \frac{L}{2}\right)$ can still take arbitrary values. As a result, the domain of the adjoint operator $\hat{p}^{\dagger}$ (which acts on $\chi$ ) is not further restricted by a boundary condition, such that $D\left(\hat{p}^{\dagger}\right) \supset D(\hat{p})$. Since $D\left(\hat{p}^{\dagger}\right) \neq D(\hat{p})$, although $\hat{p}$ is Hermitean, it is not self-adjoint in the Hilbert space $L^{2}\left(\left[-\frac{L}{2}, \frac{L}{2}\right]\right)$, and hence it does not represent the physical momentum of a particle that is strictly confined inside the box.

Let us consider a particle of mass $m$ that is limited to the finite interval $\left[-\frac{L}{2}, \frac{L}{2}\right]$ and subject to a regular potential $V(x)$. The wave function then must obey appropriate boundary conditions, which are characterized by self-adjoint extension parameters of the Hamiltonian $\hat{H}=-\frac{1}{2 m} \partial_{x}^{2}+V(x)$. Performing two partial integrations one obtains

$$
\begin{aligned}
\left\langle\hat{H}^{\dagger} \chi \mid \Psi\right\rangle= & \langle\chi \mid \hat{H} \Psi\rangle=\langle\hat{H} \chi \mid \Psi\rangle+\frac{1}{2 m}\left[\partial_{x} \chi(x)^{*} \Psi(x)\right. \\
& \left.-\chi(x)^{*} \partial_{x} \Psi(x)\right]_{-L / 2}^{L / 2} .
\end{aligned}
$$

We choose the domain $D(\hat{H})$ that contains those wave functions whose second derivative is square integrable and that obey the Robin boundary conditions

$\gamma_{+} \Psi\left(\frac{L}{2}\right)+\partial_{x} \Psi\left(\frac{L}{2}\right)=0, \gamma_{-} \Psi\left(-\frac{L}{2}\right)-\partial_{x} \Psi\left(-\frac{L}{2}\right)=0$.

Inserting these relations into the square bracket in Eq. (2), the Hermiticity condition takes the form

$$
\begin{aligned}
& {\left[\partial_{x} \chi\left(\frac{L}{2}\right)^{*}+\gamma_{+} \chi\left(\frac{L}{2}\right)^{*}\right] \Psi\left(\frac{L}{2}\right)} \\
& \quad-\left[\partial_{x} \chi\left(-\frac{L}{2}\right)^{*}-\gamma_{-} \chi\left(-\frac{L}{2}\right)^{*}\right] \Psi\left(-\frac{L}{2}\right)=0 .
\end{aligned}
$$

Since $\Psi\left( \pm \frac{L}{2}\right)$ can take arbitrary values, this implies

$\gamma_{+}^{*} \chi\left(\frac{L}{2}\right)+\partial_{x} \chi\left(\frac{L}{2}\right)=0, \quad \gamma_{-}^{*} \chi\left(-\frac{L}{2}\right)-\partial_{x} \chi\left(-\frac{L}{2}\right)=0$,

which characterizes the domain $D\left(\hat{H}^{\dagger}\right)$ of $\hat{H}^{\dagger}$ (that acts on $\chi)$. The two domains coincide, $D\left(\hat{H}^{\dagger}\right)=D(\hat{H})$, only if $\gamma_{ \pm}^{*}=$ $\gamma_{ \pm} \in \mathbb{R}$. This defines a two-parameter family of self-adjoint extensions of $\hat{H}$. The boundary conditions of Eq. (3) guarantee that the probability current

$$
j(x)=\frac{1}{2 m i}\left[\Psi(x)^{*} \partial_{x} \Psi(x)-\partial_{x} \Psi(x)^{*} \Psi(x)\right],
$$

does not leak outside the box, i.e., $j\left( \pm \frac{L}{2}\right)=0$. Not surprisingly, self-adjointness ensures probability conservation. We conclude that self-adjointness does not require the wave function to vanish at the boundary. Dirichlet boundary conditions, $\Psi\left( \pm \frac{L}{2}\right)=0$, correspond to $\gamma_{ \pm} \rightarrow \infty$, while Neumann boundary conditions, $\partial_{x} \Psi\left( \pm \frac{L}{2}\right)=0$, result from $\gamma_{ \pm}=0$. As we will see, the self-adjoint extension parameters $\gamma_{ \pm}$can be interpreted as the strengths of $\delta$-function potentials at the two boundaries.
In the absence of a potential $(V(x)=0)$ it is easy to derive the energy spectrum, $\hat{H} \psi_{l}(x)=E_{l} \psi_{l}(x), l \in \mathbb{N}$. The states with $E_{l}>0$ are given by $\psi_{l}(x)=A \exp (i k x)+B \exp (-i k x)$, with the energy quantization condition

$$
\exp (2 i k L)=\frac{\left(\gamma_{+}-i k\right)\left(\gamma_{-}-i k\right)}{\left(\gamma_{+}+i k\right)\left(\gamma_{-}+i k\right)}, \quad E_{l}=\frac{k^{2}}{2 m} .
$$

For negative values of $\gamma_{ \pm}$there are, in addition, negative energy states localized on the boundaries [18]. With general Robin boundary conditions, parity symmetry requires $\gamma_{-}=$ $\gamma_{+}$in addition to $V(-x)=V(x)$.

Since the operator $\hat{p}$ is not self-adjoint in $L^{2}\left(\left[-\frac{L}{2}, \frac{L}{2}\right]\right)$, one way to proceed is to enlarge the Hilbert space to $L^{2}(\mathbb{R})$ and to still use the standard momentum operator [24]. For this purpose, one regularizes the problem in the infrared by turning the infinite square-well potential into a finite one, with $V(x)=V_{0}$ for $|x|>\frac{L}{2}$, and finally taking the limit $V_{0} \rightarrow \infty$ [23]. When applied to a particle in a box, a measurement of the standard momentum operator projects its wave function on a momentum eigenstate $\langle x \mid k\rangle=\exp (i k x)$ and thus ejects the particle outside of the box. In the limit $V_{0} \rightarrow \infty$ one has $\langle k|V| k\rangle \rightarrow \infty$, i.e., the idealized projective momentum measurement transfers an infinite amount of energy to the particle. This is even the case for an idealized measurement with finite resolution. Obviously, actual experimental measurements consume only a finite amount of energy and are thus not completely adequately described by their idealized counterpart. The Fourier transform $\widetilde{\Psi}(k)=\int_{-L / 2}^{L / 2} d x \Psi(x) \exp (-i k x)$ of the wave function yields the probability density $\frac{1}{2 \pi}|\widetilde{\Psi}(k)|^{2}$ for measuring the standard unquantized momentum value $k \in \mathbb{R}$.

\section{A SELF-ADJOINT MOMENTUM OPERATOR FOR THE INTERVAL}

We will now develop an alternative concept for the momentum of a particle in a box, which limits itself strictly to the interior of the box, even after a momentum measurement. The question is not whether the alternative or the standard concept is the correct one, but rather which concept is appropriate in a particular physical situation. If a concrete momentum measurement ejects the particle outside of the box and yields an unquantized momentum value, the standard concept applies. If, on the other hand, the measured momentum is quantized and the particle remains inside the box after the measurement, the alternative concept is appropriate.

In contrast to the standard approach, we now regularize the problem in the ultraviolet by introducing a lattice with spacing $a$, and we finally send $a \rightarrow 0$. This will naturally lead to the doubled Hilbert space $L^{2}\left(\left[-\frac{L}{2}, \frac{L}{2}\right]\right) \times \mathbb{C}^{2}$ as the key to the solution of this long-standing problem. The subtleties associated with Hermiticity versus self-adjointness arise because the Hilbert space is infinite-dimensional. In order to gain a better understanding of the problem, we discretize it onto a finite lattice of $N$ points, such that the Hilbert space becomes $N$-dimensional [25-28]. As illustrated in Fig. 1, we divide the interval $\left[-\frac{L}{2}, \frac{L}{2}\right]$ into $N=L / a$ segments of size $a$, and introduce a lattice point in the middle of each segment, such that $x=n a, n \in\left\{-\frac{N-1}{2},-\frac{N-3}{2}, \ldots, \frac{N-3}{2}, \frac{N-1}{2}\right\}$. We choose $N$ to be odd, such that $n \in \mathbb{Z}$. The kinetic energy is then 


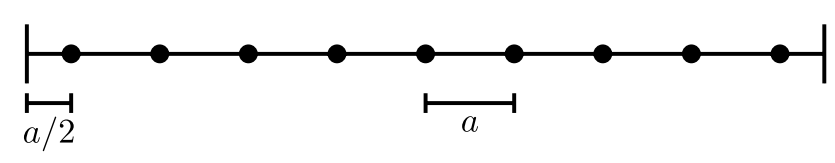

FIG. 1. Lattice with $N=9$ points in the interval $\left[-\frac{L}{2}, \frac{L}{2}\right]$.

given by a discretized second derivative, such that the $N \times N$ Hermitean matrix $\hat{H}$ takes the form

$$
\begin{aligned}
\hat{H} & =-\frac{1}{2 m a^{2}}\left(\begin{array}{rrrrrrr}
-1 & 1 & 0 & \ldots & 0 & 0 & 0 \\
1 & -2 & 1 & \ldots & 0 & 0 & 0 \\
0 & 1 & -2 & \ldots & 0 & 0 & 0 \\
\cdot & . & . & \ldots & . & . & . \\
. & . & . & \ldots & . & . & \cdot \\
0 & 0 & 0 & \ldots & -2 & 1 & 0 \\
0 & 0 & 0 & \ldots & 1 & -2 & 1 \\
0 & 0 & 0 & \ldots & 0 & 1 & -1
\end{array}\right) \\
& +\operatorname{diag}\left(V_{-(L-a) / 2}+\frac{\gamma_{-}}{2 m a}, V_{-(L-3 a) / 2}, V_{-(L-5 a) / 2}\right. \\
& \left.\ldots, V_{(L-5 a) / 2}, V_{(L-3 a) / 2}, V_{(L-a) / 2}+\frac{\gamma_{+}}{2 m a}\right) .
\end{aligned}
$$

On the lattice, the self-adjoint extension parameters $\gamma_{ \pm}$are directly incorporated into the Hamiltonian as additional terms on the diagonal, which are equivalent to $\delta$-function potentials at the boundary in the continuum limit $a \rightarrow 0$. In the absence of a potential $\left(V_{x}=0\right)$ it is straightforward to solve the Schrödinger equation $\hat{H} \psi_{l, x}=E_{l} \psi_{l, x}$ by the same ansatz as in the continuum, $\psi_{l, x}=A \exp (i k x)+B \exp (-i k x)$. The quantization condition for $E_{l}=\frac{1}{2 m}\left(\frac{2}{a} \sin \frac{k a}{2}\right)^{2}$ takes the form

$$
\begin{aligned}
\exp (2 i k(L-a))= & \frac{\gamma_{+}+\frac{1}{a}[1-\exp (i k a)]-2 m E_{l} a}{\gamma_{+}+\frac{1}{a}[1-\exp (-i k a)]-2 m E_{l} a} \\
& \times \frac{\gamma_{-}+\frac{1}{a}[1-\exp (i k a)]-2 m E_{l} a}{\gamma_{-}+\frac{1}{a}[1-\exp (-i k a)]-2 m E_{l} a} .
\end{aligned}
$$

Indeed, this reduces to Eq. (7) in the continuum limit. Similar expressions exist for negative values of $\gamma_{ \pm}$.

On the lattice the momentum operator should be represented by a discretized first derivative. It is necessary to distinguish forward and backward derivatives [29-31]

$$
\hat{p}_{F}=-\frac{i}{a}\left(\begin{array}{rrrrrrr}
-1 & 1 & 0 & \ldots & 0 & 0 & 0 \\
0 & -1 & 1 & \ldots & 0 & 0 & 0 \\
0 & 0 & -1 & \ldots & 0 & 0 & 0 \\
. & . & . & \ldots & . & . & . \\
. & . & . & \ldots & . & . & . \\
0 & 0 & 0 & \ldots & -1 & 1 & 0 \\
0 & 0 & 0 & \ldots & 0 & -1 & 1 \\
0 & 0 & 0 & \ldots & 0 & 0 & \lambda_{+}
\end{array}\right)
$$

$$
\hat{p}_{B}=-\frac{i}{a}\left(\begin{array}{ccccccc}
-\lambda_{-} & 0 & 0 & \ldots & 0 & 0 & 0 \\
-1 & 1 & 0 & \ldots & 0 & 0 & 0 \\
0 & -1 & 1 & \ldots & 0 & 0 & 0 \\
. & . & . & \ldots & . & . & . \\
. & . & . & \ldots & . & . & . \\
0 & 0 & 0 & \ldots & 1 & 0 & 0 \\
0 & 0 & 0 & \ldots & -1 & 1 & 0 \\
0 & 0 & 0 & \ldots & 0 & -1 & 1
\end{array}\right) .
$$

Just as $\gamma_{ \pm}$, the parameters $\lambda_{ \pm} \in i \mathbb{R}$ will turn into two selfadjoint extension parameters in the continuum limit. Since neither $\hat{p}_{F}$ nor $\hat{p}_{B}$ is Hermitean, we construct the Hermitean and anti-Hermitean combinations

$$
\begin{aligned}
\hat{p}_{R} & =\frac{1}{4}\left(\hat{p}_{F}+\hat{p}_{F}^{\dagger}+\hat{p}_{B}+\hat{p}_{B}^{\dagger}\right) \\
& =-\frac{i}{2 a}\left(\begin{array}{ccccccc}
-\lambda_{-} & 1 & 0 & \ldots & 0 & 0 & 0 \\
-1 & 0 & 1 & \ldots & 0 & 0 & 0 \\
0 & -1 & 0 & \ldots & 0 & 0 & 0 \\
\cdot & . & \cdot & \ldots & \cdot & \cdot & \cdot \\
. & . & . & \ldots & . & . & \cdot \\
0 & 0 & 0 & \ldots & 0 & 1 & 0 \\
0 & 0 & 0 & \ldots & -1 & 0 & 1 \\
0 & 0 & 0 & \ldots & 0 & -1 & \lambda_{+}
\end{array}\right), \\
i \hat{p}_{I} & =\frac{1}{4}\left(\hat{p}_{F}-\hat{p}_{F}^{\dagger}+\hat{p}_{B}-\hat{p}_{B}^{\dagger}\right) \\
& =\frac{i}{2 a} \operatorname{diag}(1,0,0, \ldots, 0,0,-1) .
\end{aligned}
$$

The total momentum operator $\frac{1}{2}\left(\hat{p}_{F}+\hat{p}_{B}\right)=\hat{p}_{R}+i \hat{p}_{I}$ is not Hermitean, but has a Hermitean component $\hat{p}_{R}$ and an antiHermitean component $\hat{p}_{I}$, which is diagonal in the position basis. In the continuum limit, it reduces to $\delta$-functions at the boundary $[32,33]$. The Hermitean component $\hat{p}_{R}$ results from a symmetrized forward-backward next-to-nearest neighbor derivative, which extends over two lattice spacings.

A characteristic feature of momentum is that it changes sign under parity. Hence, the operator $\hat{p}_{R}+i \hat{p}_{I}$ should anticommute with the unitary transformation $U_{P}$, with $U_{P} \Psi_{x}=$ $\Psi_{-x}$, that represents parity in Hilbert space. For $\lambda_{+}=\lambda_{-}$one indeed obtains $U_{P} \hat{p}_{F} U_{P}^{\dagger}=-\hat{p}_{B}, U_{P} \hat{p}_{B} U_{P}^{\dagger}=-\hat{p}_{F}, U_{P} \hat{p}_{R} U_{P}^{\dagger}=$ $-\hat{p}_{R}, U_{P} \hat{p}_{I} U_{P}^{\dagger}=-\hat{p}_{I}$. It is straightforward to solve the eigenvalue problem $\hat{p}_{R} \phi_{k, x}=\frac{1}{a} \sin (k a) \phi_{k, x}=\hat{k} \phi_{k, x}$. The corresponding eigenstates $\phi_{k, x}$ (with $x=n a$ ) take the form

$$
\begin{aligned}
\phi_{k, x} & =A \exp (i k x)+B \exp (-i k x), \text { for } n \text { even, } \\
\phi_{k, x} & =A \exp (i k x)-B \exp (-i k x), \text { for } n \text { odd, }
\end{aligned}
$$

and the momentum quantization condition is given by

$$
\exp (2 i k L)=\frac{\left(1+\lambda_{+} \exp (i k a)\right)\left(1-\lambda_{-} \exp (i k a)\right)}{\left(\exp (i k a)-\lambda_{+}\right)\left(\exp (i k a)+\lambda_{-}\right)} .
$$

For $\lambda_{+}=\lambda_{-}= \pm i$ this implies

$$
k=\frac{\pi n}{L}, \quad n \in\left\{-\frac{N-1}{2},-\frac{N-3}{2}, \ldots, \frac{N-3}{2}, \frac{N-1}{2}\right\} .
$$

It is important to point out that the eigenvalues $\hat{k}=\frac{1}{a} \sin (k a)$ are in one-to-one correspondence with the values of $k$ from Eq. (14), and are hence not degenerate. 
At this stage, we have reached a completely satisfactory description of the momentum operator in the $N$-dimensional Hilbert space of the $N$-point lattice. However, it is not entirely trivial to obtain an equivalent continuum description that agrees with the lattice results in the limit $a \rightarrow 0$. In this context, it is important to note that the eigenfunctions $\phi_{k, x}$ of Eq. (12) depend explicitly on whether the lattice point $x=n a$ has an even or odd value of $n$. In fact, this is the crucial insight that leads to the appropriate mathematical description also directly in the continuum. In order to describe the operator $\hat{p}_{R}$ in the continuum, it is necessary to maintain the concept of even and odd degrees of freedom. Obviously, the points $x \in \mathbb{R}$ cannot be divided into an even and an odd subset. However, it is natural to introduce a two-component wave function on which $p_{R}$ acts as a $2 \times 2$ matrix

$$
\hat{p}_{R}=-i\left(\begin{array}{cc}
0 & \partial_{x} \\
\partial_{x} & 0
\end{array}\right)=-i \sigma_{1} \partial_{x}, \quad \Psi(x)=\left(\begin{array}{l}
\Psi_{e}(x) \\
\Psi_{o}(x)
\end{array}\right) .
$$

This includes additional ultraviolet degrees of freedom which are necessary for the self-adjointness of the momentum operator, but not of the Hamiltonian. By partial integration we obtain

$$
\begin{aligned}
\left\langle\hat{p}_{R}^{\dagger} \chi \mid \Psi\right\rangle & =\left\langle\chi \mid \hat{p}_{R} \Psi\right\rangle \\
& =\left\langle\hat{p}_{R} \chi \mid \Psi\right\rangle-i\left[\chi_{e}(x)^{*} \Psi_{o}(x)+\chi_{o}(x)^{*} \Psi_{e}(x)\right]_{-L / 2}^{L / 2} .
\end{aligned}
$$

We now impose the boundary conditions

$$
\Psi_{o}\left(\frac{L}{2}\right)=\lambda_{+} \Psi_{e}\left(\frac{L}{2}\right), \quad \Psi_{o}\left(-\frac{L}{2}\right)=\lambda_{-} \Psi_{e}\left(-\frac{L}{2}\right),
$$

which define the domain $D\left(\hat{p}_{R}\right)$. Inserting these relations in the square bracket in Eq. (16), the Hermiticity condition takes the form

$$
\begin{aligned}
& {\left[\chi_{e}\left(\frac{L}{2}\right)^{*} \lambda_{+}+\chi_{o}\left(\frac{L}{2}\right)^{*}\right] \Psi_{e}\left(\frac{L}{2}\right)} \\
& \quad-\left[\chi_{e}\left(-\frac{L}{2}\right)^{*} \lambda_{-}+\chi_{o}\left(-\frac{L}{2}\right)^{*}\right] \Psi_{e}\left(-\frac{L}{2}\right)=0 .
\end{aligned}
$$

Since $\Psi_{e}\left( \pm \frac{L}{2}\right)$ can take arbitrary values, this implies

$$
\chi_{o}\left(\frac{L}{2}\right)=-\lambda_{+}^{*} \chi_{e}\left(\frac{L}{2}\right), \quad \chi_{o}\left(-\frac{L}{2}\right)=-\lambda_{-}^{*} \chi_{e}\left(-\frac{L}{2}\right) .
$$

Self-adjointness of $\hat{p}_{R}$ requires $D\left(\hat{p}_{R}^{\dagger}\right)=D\left(\hat{p}_{R}\right)$, which implies $\lambda_{ \pm}=-\lambda_{ \pm}^{*}$ such that $\lambda_{ \pm} \in i \mathbb{R}$. Hence, there is a two-parameter family of self-adjoint extensions, characterized by the purely imaginary parameters $\lambda_{+}$and $\lambda_{-}$. Parity symmetry maps the two boundaries onto each other and implies $\lambda_{+}=\lambda_{-}$.

Let us now consider the eigenvalue problem of the selfadjoint operator $\hat{p}_{R}$. In analogy to Eq. (12) on the lattice, we make the ansatz

$$
\phi_{k}(x)=\left(\begin{array}{l}
A \exp (i k x)+B \exp (-i k x) \\
A \exp (i k x)-B \exp (-i k x)
\end{array}\right) .
$$

Imposing the boundary conditions implies

$$
\left(\begin{array}{cc}
\left(1-\lambda_{+}\right) \exp (i k L) & -\left(1+\lambda_{+}\right) \\
\left(1-\lambda_{-}\right) \exp (-i k L) & -\left(1+\lambda_{-}\right)
\end{array}\right)\left(\begin{array}{l}
A \\
B
\end{array}\right)=0 .
$$

A nontrivial solution arises only when the determinant of the matrix vanishes

$$
\exp (2 i k L)=\frac{\left(1+\lambda_{+}\right)\left(1-\lambda_{-}\right)}{\left(1-\lambda_{+}\right)\left(1+\lambda_{-}\right)} .
$$

This agrees with the lattice momentum quantization condition of Eq. (13) in the continuum limit $a \rightarrow 0$. Hence, the two-component formulation of Eq. (15) indeed provides the correct continuum description of the momentum operator $\hat{p}_{R}$ that was constructed on the lattice in Eq. (11).

\section{EMBEDDING THE HAMILTONIAN}

Next we consider the Hamiltonian

$$
\hat{H}(\mu)=\left(\begin{array}{cc}
-\frac{1}{2 m} \partial_{x}^{2}+V(x) & 0 \\
0 & -\frac{1}{2 m} \partial_{x}^{2}+V(x)
\end{array}\right)+\mu \hat{P}_{-} .
$$

Here $\hat{P}_{-}$is a projection operator on states $\Psi^{-}(x)$ with $\Psi_{o}^{-}(x)=-\Psi_{e}^{-}(x)$. On the lattice, such states have energies at the cutoff scale $1 / a$. For $\mu \rightarrow \infty$ the corresponding ultraviolet degrees of freedom are removed from the energy spectrum of the continuum theory as well. The operator $\hat{P}_{+}$projects on the remaining finite-energy states $\Psi^{+}(x)$ with $\Psi_{o}^{+}(x)=\Psi_{e}^{+}(x)$, i.e.,

$$
\begin{aligned}
& \hat{P}_{ \pm}=\frac{1}{2}\left(\begin{array}{cc}
1 & \pm 1 \\
\pm 1 & 1
\end{array}\right), \quad \hat{P}_{ \pm}^{2}=\hat{P}_{ \pm}, \hat{P}_{+} \hat{P}_{-}=\hat{P}_{-} \hat{P}_{+}=0 \\
& \hat{P}_{+}+\hat{P}_{-}=\mathbb{1}, \quad \Psi^{ \pm}(x)=P_{ \pm} \Psi(x) .
\end{aligned}
$$

Next we introduce the boundary conditions

$$
\left(\begin{array}{c}
\Psi_{o}\left( \pm \frac{L}{2}\right) \\
\partial_{x} \Psi_{o}\left( \pm \frac{L}{2}\right)
\end{array}\right)=e^{i \theta_{ \pm}}\left(\begin{array}{cc}
a_{ \pm} & \pm b_{ \pm} \\
\pm c_{ \pm} & d_{ \pm}
\end{array}\right)\left(\begin{array}{c}
\Psi_{e}\left( \pm \frac{L}{2}\right) \\
\partial_{x} \Psi_{e}\left( \pm \frac{L}{2}\right)
\end{array}\right) .
$$

Again demanding that $j\left( \pm \frac{L}{2}\right)=0$, it is easy to derive the conditions $a_{ \pm}, b_{ \pm}, c_{ \pm}, d_{ \pm} \in \mathbb{R}$ and $a_{ \pm} d_{ \pm}-b_{ \pm} c_{ \pm}=-1$. Together with $\theta_{ \pm}$, this defines a family of self-adjoint extensions with eight independent parameters [34]. However, we are not interested in the most general Hamiltonian in this class. We just need to find an appropriate continuum formulation for the original Hamiltonian in the Hilbert space of two-component wave functions, which is essential for describing the momentum operator $\hat{p}_{R}$. Since the boundary conditions should support the finite-energy states with $\Psi_{o}^{+}(x)=\Psi_{e}^{+}(x)$, this implies

$$
e^{i \theta_{ \pm}}=1, \quad a_{ \pm}=1, \quad b_{ \pm}=0, \quad d_{ \pm}=-1 .
$$

Using the parameters of Eq. (26), Eq. (25) reduces to

$$
-\frac{c_{ \pm}}{2} \Psi^{+}\left( \pm \frac{L}{2}\right) \pm \partial_{x} \Psi^{+}\left( \pm \frac{L}{2}\right)=0, \quad \Psi^{-}\left( \pm \frac{L}{2}\right)=0 .
$$

When we identify $\Psi^{+}(x)$ with the wave functions in the original Hilbert space and set $\gamma_{ \pm}=-c_{ \pm} / 2$, this is equivalent to 
the Robin boundary conditions of Eq. (3). Interestingly, the wave functions $\Psi^{-}(x)$ obey Dirichlet boundary conditions. It is important to note that the Hamiltonian $\hat{H}(\mu)$ and the momentum operator $\hat{p}_{R}$ are not defined in the same domains, i.e., $D(\hat{H}(\mu)) \neq D\left(\hat{p}_{R}\right)$. Since $\lambda_{ \pm} \in i \mathbb{R}$, Eq. (17) is indeed incompatible with the boundary conditions associated with Eq. (26). This implies that an energy eigenstate is a superposition of infinitely many momentum eigenstates. Furthermore, although at the formal level of differential expressions the kinetic energy $\hat{T}=-\frac{1}{2 m} \partial_{x}^{2}$ and the momentum $\hat{p}_{R}=-i \sigma_{1} \partial_{x}$ seem to commute, in fact $\left[\hat{T}, \hat{p}_{R}\right] \neq 0$ due to domain incompatibilities [11]. An exception are Dirichlet boundary conditions, $-c_{ \pm} \rightarrow$ $\infty$, since for them $\Psi_{e}\left( \pm \frac{L}{2}\right)=\Psi_{o}\left( \pm \frac{L}{2}\right)=0$.

\section{ALTERNATIVE MOMENTUM MEASUREMENTS FOR A PARTICLE IN A BOX}

Let us apply the alternative concept to the question of momentum measurements for a particle that is confined inside a box. We assume parity symmetry such that $\lambda_{+}=\lambda_{-}=\lambda$. The corresponding momentum eigenvalues and eigenfunctions are

$$
\begin{aligned}
\hat{p}_{R} \phi_{k}(x) & =k \phi_{k}(x), k=\frac{\pi n}{L}, n \in \mathbb{Z}, \sigma=\frac{1-\lambda}{1+\lambda} \in U(1) \\
\phi_{k}(x) & =\frac{1}{2 \sqrt{L}}\left(\begin{array}{l}
\exp (i k x)+\sigma \exp (-i k x) \\
\exp (i k x)-\sigma \exp (-i k x)
\end{array}\right), \quad n \text { even, } \\
\phi_{k}(x) & =\frac{1}{2 \sqrt{L}}\left(\begin{array}{l}
\exp (i k x)-\sigma \exp (-i k x) \\
\exp (i k x)+\sigma \exp (-i k x)
\end{array}\right), \quad n \text { odd. }
\end{aligned}
$$

After a momentum measurement, the particle is in an eigenstate $\phi_{k}(x)$, with both a $\phi_{k}^{+}(x)$ and $\phi_{k}^{-}(x)$ component. The energy of the $\phi_{k}^{-}(x)$ component diverges for $\mu \rightarrow \infty$. This implies that a momentum measurement transfers an infinite amount of energy to the particle. The same happens in a standard momentum measurement on a particle that moves along the entire real axis in a potential that diverges at infinity, $V( \pm \infty) \rightarrow \infty$.

Independent of $\lambda$, when projected onto the finite-energy sector, the momentum eigenstates are just $\phi_{k, e}^{+}(x)=\phi_{k, o}^{+}(x)=$ $\frac{1}{2 \sqrt{L}} \exp (i k x)$. The amplitude to measure the discrete value $k=\pi n / L$ of the alternative momentum in a general lowenergy state $\frac{1}{\sqrt{2}}\left(\begin{array}{l}\Psi(x) \\ \Psi(x)\end{array}\right)$ is

$$
\frac{1}{\sqrt{2 L}} \int_{-L / 2}^{L / 2} d x \Psi(x) \exp (-i k x)=\frac{1}{\sqrt{2 L}} \widetilde{\Psi}(k)
$$

This is exactly $\sqrt{\pi / L}$ times the probability amplitude $\frac{1}{\sqrt{2 \pi}} \widetilde{\Psi}(k)$ to measure the standard unquantized momentum $k \in \mathbb{R}$. Hence the results of both types of momentum measurements are closely related (cf. Fig. 2), despite the fact that the states after the measurement are radically different. In particular, a standard momentum measurement ejects the particle from the box, while the alternative measurement leaves it inside.

Putting $V(x)=0$, we finally consider the standard textbook case with Dirichlet boundary conditions, $\Psi\left( \pm \frac{L}{2}\right)=0$,

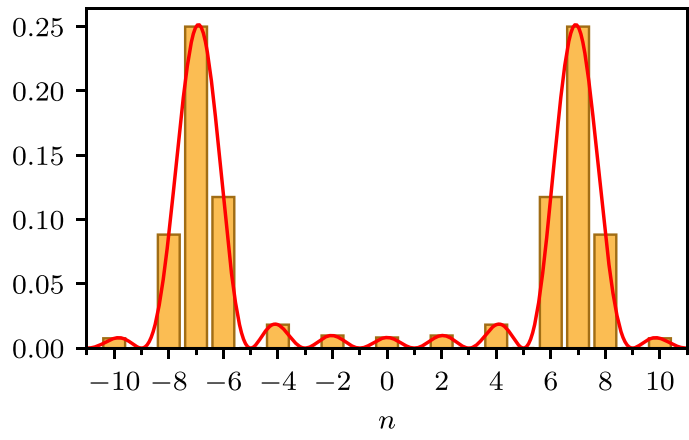

FIG. 2. Probability to measure the quantized momentum $k=$ $\pi n / L$ in the energy eigenstate $\psi_{l}(x)$ with $l=7$, as a function of $n$, compared to $\pi / L$ times the probability density $\frac{1}{2 \pi}\left|\widetilde{\psi}_{l}(k)\right|^{2}$ to measure the unquantized standard momentum $k \in \mathbb{R}$.

which correspond to $\gamma_{+}=\gamma_{-} \rightarrow \infty$, and

$$
\begin{aligned}
& \hat{H}(\mu) \psi_{l}(x)=E_{l} \psi_{l}(x), \quad E_{l}=\frac{\pi^{2} l^{2}}{2 m L^{2}}, \quad l \in \mathbb{N}_{>0} \\
& \psi_{l}(x)=\frac{1}{\sqrt{L}}\left(\begin{array}{l}
\cos (\pi l x / L) \\
\cos (\pi l x / L)
\end{array}\right), \quad l \text { odd, } \\
& \psi_{l}(x)=\frac{1}{\sqrt{L}}\left(\begin{array}{l}
\sin (\pi l x / L) \\
\sin (\pi l x / L)
\end{array}\right), \quad l \text { even. }
\end{aligned}
$$

In this case, one obtains $\left\langle\psi_{l}\left|\hat{p}_{R}\right| \psi_{l}\right\rangle=\left\langle\psi_{l}\left|\hat{p}_{I}\right| \psi_{l}\right\rangle=0$. As illustrated in Fig. 2, when one measures the momentum $\hat{p}_{R}$ in an energy eigenstate $\psi_{l}(x)$, one obtains $k= \pm \pi l / L$ each with probability $1 / 4$. The probability to measure $k=\pi n / L$ for $n \neq \pm l$ is $\left|\left\langle\phi_{k} \mid \psi_{l}\right\rangle\right|^{2}=4 l^{2} /\left(\pi\left(l^{2}-n^{2}\right)\right)^{2}$ if $(-1)^{n}=-(-1)^{l}$ and zero otherwise.

\section{CONCLUSIONS}

Complementary to the operator $\hat{p}=-i \partial_{x}$ that acts in $L^{2}(\mathbb{R})$, the alternative concept for the momentum $\hat{p}_{R}+i \hat{p}_{I}$ of a particle that is strictly confined inside a box, operates in the doubled Hilbert space $L^{2}\left(\left[-\frac{L}{2}, \frac{L}{2}\right]\right) \times \mathbb{C}^{2}$. Both idealized momentum measurements transfer an infinite energy to the particle, the former in the infrared and the latter in the ultraviolet. The two alternative momentum concepts are both mathematically well founded because they are associated with self-adjoint operators acting in two different Hilbert spaces. The physical conditions of a particular experimental momentum measurement must decide which concept of an idealized projective momentum measurement is appropriate. For example, if one switches off an optical box potential that confined an ultracold atomic gas before a momentum measurement is performed, all of space becomes available to the atoms after the measurement, and the standard momentum concept with unquantized momentum values applies. If, on the other hand, the confining potential is maintained during a momentum measurement, momentum is quantized and the alternative concept applies.

It is easy to generalize $\hat{p}_{R}$ to higher dimensions $\hat{\vec{p}}_{R}=$ $-i \sigma_{1} \vec{\nabla}, \Psi_{o}(\vec{x})=\lambda(\vec{x}) \Psi_{e}(\vec{x}), \lambda(\vec{x}) \in i \mathbb{R}, \vec{x} \in \partial \Omega, \Omega \in \mathbb{R}^{d}$. The alternative momentum concept enables canonical quantization in a bounded region of space [11], it provides a physical 
interpretation for the uncertainty relation [35-39] in a finite volume [18], and, in contrast to the standard momentum [40,41], it satisfies the Ehrenfest theorem [42] in a box [43]. Indeed, the alternative momentum concept has far-reaching consequences and promising applications in different areas of physics.

\section{ACKNOWLEDGMENTS}

We thank I. Albrecht, M. Blau, J. Herrmann, C. Tretter, and V. Wyss for very interesting discussions. The research of M.H.A. is supported by M. Müller. U.J.W. is supported by the Schweizerischer Nationalfonds.
[1] A. L. Gaunt, T. F. Schmidutz, I. Gotlibovych, R. P. Smith, and Z. Hadzibabic, Bose-Einstein Condensation of Atoms in a Uniform Potential, Phys. Rev. Lett. 110, 200406 (2013).

[2] P. Harrison, Quantum Wells, Wires and Dots (Wiley, New York, 2005).

[3] P. Seba, Wave Chaos in Singular Quantum Billiard, Phys. Rev. Lett. 64, 1855 (1990).

[4] L. A. Ponomarenko, F. Schedin, M. I. Katsnelson, R. Yang, E. W. Hill, K. S. Novoselov, and A. K. Geim, Chaotic Dirac billiard in graphene quantum dots, Science 320, 356 (2008).

[5] A. Chodos, R. L. Jaffe, K. Johnson, C. B. Thorn, and V. F. Weisskopf, New extended model of hadrons, Phys. Rev. D 9, 3471 (1974).

[6] A. Chodos, R. L. Jaffe, K. Johnson, and C. B. Thorn, Baryon structure in the bag theory, Phys. Rev. D 10, 2599 (1974).

[7] P. Hasenfratz and J. Kuti, The quark bag model, Phys. Rep. 40, 75 (1978).

[8] D. B. Kaplan, A method for simulating chiral fermions on the lattice, Phys. Lett. B 288, 342 (1992).

[9] Y. Shamir, Chiral fermions from lattice boundaries, Nucl. Phys. B 406, 90 (1993).

[10] L. Randall and R. Sundrum, Large Mass Hierarchy from a Small Extra Dimension, Phys. Rev. Lett. 83, 3370 (1999),

[11] M. Al-Hashimi and U.-J. Wiese, Canonical quantization on the half-line and in an interval based upon a new concept for the momentum in a space with boundaries, Phys. Rev. Research 3, 033079 (2021).

[12] J. von Neumann, Mathematische Grundlagen der Quantenmechanik (Springer, Berlin, 1932).

[13] M. Reed and B. Simon, Methods of Modern Mathematical Physics II, Fourier Analysis, Self-Adjointness (Academic Press, New York, 1975).

[14] F. Gieres, Mathematical surprises and Dirac's formalism in quantum mechanics, Rep. Prog. Phys. 63, 1893 (2000).

[15] T. Jurić, Observables in Quantum Mechanics and the importance of self-adjointness, arXiv:2103.01080.

[16] R. Balian and C. Bloch, Distribution of eigenfrequencies for the wave equation in a finite domain: I. Three-dimensional problem with smooth boundary surface, Ann. Phys. 60, 401 (1970).

[17] M. Carreau, E. Farhi, and S. Gutmann, Functional integral for a free particle in a box, Phys. Rev. D 42, 1194 (1990).

[18] M. H. Al-Hashimi and U.-J. Wiese, From a particle in a box to the uncertainty relation in a quantum dot and to reflecting walls for relativistic fermions, Ann. Phys. 327, 2742 (2012).

[19] G. Bonneau, J. Faraut, and G. Valent, Self-adjoint extensions of operators and the teaching of quantum mechanics, Am. J. Phys. 69, 322 (2001).

[20] D. L. Aronstein and C. R. Stroud, Jr., Fractional wave-function revivals in the infinite square well, Phys. Rev. A 55, 4526 (1997).
[21] B. Hu, B. Li, J. Liu, and Y. Gu, Quantum Chaos of a Kicked Particle in an Infinite Potential Well, Phys. Rev. Lett. 82, 4224 (1999).

[22] F. Gori, D. Ambrosini, R. Borghi, V. Mussi, and M. Santarsiero, The propagator for a particle in a well, Eur. J. Phys. 22, 53 (2001).

[23] P. Garbaczewski and W. Karwowski, Impenetrable barriers and canonical quantization, Am. J. Phys. 72, 924 (2004).

[24] C. Cohen-Tannoudji, B. Dui, and F. Laloë, Quantum Mechanics I (Wiley, New York, 1977).

[25] T. S. Santhanam and A. R. Tekumalla, Quantum mechanics in finite dimensions, Found. Phys. 6, 583 (1976).

[26] R. Jagannathan, T. S. Santhanam, and R. Vasudevan, Finitedimensional quantum mechanics of a particle, Int. J. Theor Phys. 20, 755 (1981).

[27] R. Jagannathan and T. S. Santhanam, Finite-dimensional quantum mechanics of a particle. II, Int. J. Theor. Phys. 21, 351 (1982).

[28] A. C. de la Torre and D. Goyeneche, Quantum mechanics in finite-dimensional Hilbert space, Am. J. Phys. 71, 49 (2003).

[29] A. Martinez-Pérez and G. Torres-Vega, Translations in quantum mechanics revisited. The point spectrum case, Can. J. Phys. 94, 1365 (2016).

[30] A. Martinez-Pérez and G. Torres-Vega, Exact finite differences for quantum mechanics, IntechOpen, http://dx.doi.org/10.5772/ intechopen.71956.

[31] A. Martinez-Pérez and G. Torres-Vega, Matrices which are discrete versions of linear operations, IntechOpen, http://dx.doi. org/10.5772/intechopen.74356.

[32] P. D. Robinson and J. O. Hirschfelder, Generalized momentum operators in quantum mechanics, J. Math. Phys. 4, 338 (1963).

[33] P. D. Robinson, Integral forms for quantum-mechanical momentum operators, J. Math. Phys. 7, 2060 (1966).

[34] P. E. T. Jorgensen, S. Pedersen, and F. Tian, Momentum operators in two intervals: Spectra and phase transition, Complex Anal. Oper. Theory 7, 1735 (2013).

[35] W. Heisenberg, Über den anschaulichen Inhalt der quantentheoretischen Kinematik und Mechanik, Z. Phys. 43, 172 (1927).

[36] E. H. Kennard, Zur Quantenmechanik einfacher Bewegungstypen, Z. Phys. 44, 326 (1927).

[37] H. Weyl, Gruppentheorie und Quantenmechanik (Hirzel, Leipzig, 1928).

[38] H. P. Robertson, The uncertainty principle, Phys. Rev. 34, 163 (1929).

[39] E. Schrödinger, Zum heisenbergschen Unschärfeprinzip, Sitzungsberichte der Preussischen Akademie der Wissenschaften, Physikalisch-mathematische Klasse 14, 296 (1930). 
[40] V. Alonso, S. De Vincenzo, L. Gonzalez-Diaz, On the Ehrenfest theorem in a one-dimensional box, Il Nuovo Cimento B115, 155 (2000).

[41] V. Alonso, S. De Vincenzo, and L. Gonzalez-Diaz, Ehrenfest's theorem and Bohm's quantum potential in a one-dimensional box, Phys. Lett. A 287, 23 (2001).
[42] P. Ehrenfest, Bemerkung über die angenäherte Gültigkeit der klassischen Mechanik innerhalb der Quantenmechanik, Z. Phys. 45, 455 (1927).

[43] I. Albrecht, J. Herrmann, U.-J. Wiese, and V. Wyss (unpublished). 